Amphibia-Reptilia

\title{
COI-based barcoding of Chinese vipers (Reptilia: Squamata: Viperidae)
}

\section{Qin Liu ${ }^{1,2}$, Fei Zhu ${ }^{1,2}$, Guanghui Zhong ${ }^{1,3}$, Yunyu Wang ${ }^{4}$, Min Fang ${ }^{1}$, Rong Xiao ${ }^{1}$, Yansen Cai ${ }^{5}$, Peng Guo ${ }^{1, *}$}

${ }^{1}$ College of Life Sciences and Food Engineering, Yibin University, Yibin 644007, China

${ }^{2}$ College of Life Sciences, Sichuan University, Chengdu 610064, China

${ }^{3}$ College of Tourism and Urban-Rural Planning, Chengdu University of Technology, Chengdu 610059, China

${ }^{4}$ State Key Laboratory of Genetic Resources and Evolution, Kunming Institute of Zoology, Chinese Academy of Sciences, Kunming, 650223, China

${ }^{5}$ Department of Medical Biology and Genetics, Luzhou Medical College, Luzhou, 646000, China

\section{Supplementary Material}

Table S1. List of specimens with localities. GP: author's personal catalogue; KIZ: Kunming Institute of Zoology, the Chinese Academy of Sciences; IEBR: Institute of Ecology and Biological Resources, Hanoi; ZFMK: Zoologisches Forschungsinstitut und Museum Alexander Koenig, Bonn. \# indicates the sequences were retrieved from GenBank. 


\begin{tabular}{|c|c|c|c|c|}
\hline Azemiops feae & GP616 & KP403569 & BOLD:ACV0634 & Anxian, Sichuan \\
\hline Azemiops feae & GP617 & KP403570 & BOLD:ACV0634 & Anxian, Sichuan \\
\hline Daboia siamensis & GP1 & KP403682 & BOLD:AAI9655 & Guangxi \\
\hline Daboia siamensis & GP2 & KP403683 & BOLD:AAI9655 & Thailand \\
\hline Daboia siamensis & S-04 & KF698971\# & - & Jiangxi \\
\hline Deinagkistrodon acutus & GP101 & KP403581 & BOLD:ACC5654 & Chongqing \\
\hline Deinagkistrodon acutus & GP102 & KP403582 & BOLD:ACC5654 & Chongqing \\
\hline Deinagkistrodon acutus & GP103 & KP403583 & BOLD:ACC5654 & Chongqing \\
\hline Deinagkistrodon acutus & GP184 & KP403584 & BOLD:ACC5654 & Guilin, Guangxi \\
\hline Deinagkistrodon acutus & GP482 & KP403585 & BOLD:AAE7483 & Huangshan, Anhui \\
\hline Gloydius brevicaudus & GP52 & KP403587 & BOLD:ACV0124 & Huangshan, Anhui \\
\hline Gloydius brevicaudus & GP53 & KP403588 & BOLD:ACV0124 & Huangshan, Anhui \\
\hline Gloydius brevicaudus & GP195 & KP403589 & BOLD:ACB4072 & Huanren, Liaoning \\
\hline Gloydius brevicaudus & GP628 & KP403590 & BOLD:ACB4072 & Kuandian, Liaoning \\
\hline Gloydius brevicaudus & GP1027 & KP403591 & BOLD:ACV0124 & South of China \\
\hline Gloydius brevicaudus & GP1099 & KP403592 & BOLD:ACB4072 & Dalian, Liaoning \\
\hline Gloydius brevicaudus & GP1480 & KP403593 & BOLD:ACB4072 & Xinlong, Hebei \\
\hline Gloydius brevicaudus & NIBRRP0000100264 & JQ798883\# & - & Sinan-gun,Korea \\
\hline Gloydius brevicaudus & NIBRRP0000100276 & JQ798884\# & - & Sinan-gun,Korea \\
\hline Gloydius brevicaudus & NIBRRP0000100265 & JQ798885\# & - & Sinan-gun,Korea \\
\hline
\end{tabular}




\begin{tabular}{|c|c|c|c|c|}
\hline Gloydius brevicaudus & ZB-8 & KC841452\# & - & Yongzhou, Hunan \\
\hline Gloydius brevicaudus & ZB-9 & KC841453\# & - & Yongzhou, Hunan \\
\hline Gloydius intermedius & GP465 & KP403595 & BOLD:AAC6073 & Xinjiang \\
\hline Gloydius intermedius & GP3073 & KP403597 & BOLD:ACV1406 & Yan'an, Shaanxi \\
\hline Gloydius lijianlii & GP1162 & KP403608 & BOLD:ACV0199 & Yantai, Shandong \\
\hline Gloydius lijianlii & GP1163 & KP403609 & BOLD:ACV0199 & Yantai, Shandong \\
\hline Gloydius liupanensis & GP198 & KP403598 & BOLD:ACV0723 & Liupanshan, Ningxia \\
\hline Gloydius liupanensis & GP206 & KP403599 & BOLD:ACV0723 & Liupanshan, Ningxia \\
\hline Gloydius liupanensis & GP215 & KP403600 & BOLD:ACV0723 & Liupanshan, Ningxia \\
\hline Gloydius qinlingensis & GP197 & KP403601 & BOLD:ACV0428 & Zhouzhi, Shaanxi \\
\hline Gloydius qinlingensis & GP3076 & KP403602 & BOLD:ACV0428 & Zhouzhi, Shaanxi \\
\hline Gloydius qinlingensis & GP3077 & KP403603 & BOLD:ACV0428 & Zhouzhi, Shaanxi \\
\hline Gloydius saxatilis & GP629 & KP403607 & BOLD:AAA8329 & Kuandian, Liaoning \\
\hline Gloydius saxatilis & GP1328 & KP403611 & BOLD:AAA8329 & Ji'an,Jilin \\
\hline Gloydius saxatilis & GP1329 & KP403612 & BOLD:AAA8329 & Ji'an,Jilin \\
\hline Gloydius saxatilis & GP1330 & KP403613 & BOLD:AAA8329 & Tonghua, Jilin \\
\hline Gloydius saxatilis & GP1350 & KP403614 & BOLD:AAA8329 & Huanren, Liaoning \\
\hline Gloydius saxatilis & NIBRRP0000100251 & JQ798881\# & - & Hwaseongsi, Korea \\
\hline Gloydius saxatilis & NIBRRP0000100199 & JQ798882\# & - & Samcheok-si, Korea \\
\hline Gloydius shedaoensis & GP1116 & KP403615 & BOLD:ACV0838 & Dalian, Liaoning \\
\hline Gloydius shedaoensis & GP1119 & KP403616 & BOLD:ACV0838 & Dalian, Liaoning \\
\hline Gloydius shedaoensis & GP1132 & KP403617 & BOLD:ACV0838 & Dalian, Liaoning \\
\hline Gloydius strauchi & GP106 & KP403618 & BOLD:ACV0602 & Ruoergai, Sichuan \\
\hline
\end{tabular}




\begin{tabular}{|c|c|c|c|c|}
\hline Gloydius strauchi & GP175 & KP403619 & BOLD:ACV0983 & Heishui, Sichuan \\
\hline Gloydius strauchi & GP2322 & KP403620 & BOLD:ACV0603 & Shiqu, Sichuan \\
\hline Gloydius strauchi & GP2323 & KP403621 & BOLD:ACV0603 & Shiqu, Sichuan \\
\hline Gloydius strauchi & GP2324 & KP403622 & BOLD:ACV0603 & Shiqu, Sichuan \\
\hline Gloydius ussuriensis & GP194 & KP403624 & BOLD:AAA8331 & Huanren, Liaoning \\
\hline Gloydius ussuriensis & GP1319 & KP403625 & BOLD:AAA8331 & Huanren, Liaoning \\
\hline Gloydius ussuriensis & GP1326 & KP403626 & BOLD:AAA8331 & Ji'an, Jilin \\
\hline Gloydius ussuriensis & GP1331 & KP403628 & BOLD:AAA8331 & Tonghua, Jilin \\
\hline Gloydius ussuriensis & GP1332 & KP403629 & BOLD:AAA8331 & Tonghua, Jilin \\
\hline Gloydius ussurensis & GP1333 & KP403630 & BOLD:AAA8331 & Tonghua, Jilin \\
\hline Gloydius ussurensis & NIBRRP0000100352 & JQ798886\# & - & Sacheon-si, Korea \\
\hline Gloydius ussurensis & NIBRRP0000100367 & JQ798887\# & - & Incheon, Korea \\
\hline Himalayophis tibetanus & GP149 & KP403631 & BOLD:ACV0059 & Zhangmu, Xizang \\
\hline Ovophis makazayazaya & GP343 & KP403635 & BOLD:ACU9980 & Shimen, Hunan \\
\hline Ovophis makazayazaya & GP1083 & KP403636 & BOLD:ACU9981 & Changning, Sichuan \\
\hline Ovophis makazayazaya & GP3086 & KP403637 & BOLD:ACU9981 & Shiyan, Hubei \\
\hline Ovophis tonkinensis & GP1632 & KP403638 & BOLD:ACV1715 & Maoming, Guangdong \\
\hline Ovophis tonkinensis & GP2051 & KP403639 & BOLD:ACV1716 & Longzhou, Guangdong \\
\hline Ovophis tonkinensis & GP2140 & KP403640 & BOLD:ACV0860 & Diaoluoshan, Hainan \\
\hline Ovophis zayuensis & GP89 & KP403641 & BOLD:ACV0859 & Chayu, Xizang \\
\hline Ovophis zayuensis & GP91 & KP403642 & BOLD:ACV0859 & Chayu, Xizang \\
\hline Ovophis zayuensis & GP557 & KP403643 & BOLD:ACV0859 & Pailong, Xizang \\
\hline Popeia popeiorum & GP3326 & KP403685 & BOLD:ACS7021 & Jinghong, Yunnan \\
\hline Popeia popeiorum & GP3327 & KP403686 & BOLD:ACS7021 & Jinghong, Yunnan \\
\hline
\end{tabular}




\begin{tabular}{|c|c|c|c|c|}
\hline Popeia popeiorum & GP3329 & KP403687 & BOLD:ACS7021 & Jinghong, Yunnan \\
\hline Popeia popeiorum & GP3330 & KP403688 & BOLD:ACS7021 & Jinghong, Yunnan \\
\hline Popeia popeiorum & GP3332 & KP403690 & BOLD:ACS7021 & Jinghong, Yunnan \\
\hline Popeia popeiorum & GP3334 & KP403692 & BOLD:ACS7021 & Jinghong, Yunnan \\
\hline Popeia popeiorum & GP3335 & KP403693 & BOLD:ACS7021 & Jinghong, Yunnan \\
\hline Popeia popeiorum & GP3336 & KP403694 & BOLD:ACS7021 & Jinghong, Yunnan \\
\hline Popeia popeiorum & KIZ01541 & KP403696 & BOLD:ACS7021 & Mengla, Yunnan \\
\hline Protobothrops cornutus & IEBR A2014.28/CB 2011.2 & KP403644 & BOLD:ACV0852 & Cao Bang, Vietnam \\
\hline Protobothrops cornutus & $\begin{array}{l}\text { IEBR A2014.31/PNKB } \\
2011.300\end{array}$ & KP403645 & BOLD:ACV0853 & Quang Binh, Vietnam \\
\hline Protobothrops dabieshanensis & GP2631 & KP403646 & BOLD:ACU8513 & Yuexi, Anhui \\
\hline Protobothrops himalayanus & GP3163 & KP403647 & BOLD:ACV1404 & Jilong, Xizang \\
\hline Protobothrops jerdonii & GP345 & KP403651 & BOLD:ACV0945 & Shimen, Hunan \\
\hline Protobothrops jerdonii & GP346 & KP403652 & BOLD:ACV0945 & Shimen, Hunan \\
\hline Protobothrops jerdonii & GP853 & KP403653 & BOLD:ACV0944 & Foping,Shaanxi \\
\hline Protobothrops jerdonii & GP855 & KP403654 & BOLD:ACV0944 & Foping,Shaanxi \\
\hline Protobothrops jerdonii & GP1084 & KP403655 & BOLD:ACU9909 & Henan \\
\hline Protobothrops jerdonii & GP1085 & KP403656 & BOLD:ACU9909 & Henan \\
\hline Protobothrops jerdonii & ZL-PV-111111 & JX233624\# & - & Hunan \\
\hline Protobothrops kaulbacki & GP112 & KP403657 & BOLD:ACV0904 & Motuo, Xizang \\
\hline Protobothrops kaulbacki & GP550 & KP403658 & BOLD:ACV0904 & Linzhi, Xizang \\
\hline Protobothrops mangshanensis & GP17 & KP403659 & BOLD:ACH4986 & Yizhang, Hunan \\
\hline Protobothrops mangshanensis & $\mathrm{H} 1$ & JX233619\# & - & Guangdong \\
\hline
\end{tabular}




\begin{tabular}{|c|c|c|c|c|}
\hline Protobothrops maolanensis & GP1883 & KP403660 & BOLD:ACU8512 & Libo, Guizhou \\
\hline Protobothrops maolanensis & GP1884 & KP403661 & BOLD:ACU8512 & Libo, Guizhou \\
\hline Protobothrops maolanensis & GP1903 & KP403663 & BOLD:ACU8512 & Libo, Guizhou \\
\hline Protobothrops mucrosquamatus & GP31 & KP403665 & BOLD:ACV1327 & Hongya,Sichuan \\
\hline Protobothrops mucrosquamatus & GP32 & KP403666 & BOLD:ACV1327 & Hongya,Sichuan \\
\hline Protobothrops mucrosquamatus & GP134 & KP403667 & BOLD:ACV1327 & Tongjiang, Sichuan \\
\hline Protobothrops mucrosquamatus & GP402 & KP403669 & BOLD:ACH9701 & Ruyuan, Guangdong \\
\hline Protobothrops mucrosquamatus & GP997 & KP403670 & BOLD:ACH9702 & Cenxi, Guangxi \\
\hline Protobothrops mucrosquamatus & GP1043 & KP403671 & BOLD:ACV1327 & Luxi, Hunan \\
\hline Protobothrops mucrosquamatus & GP2472 & KP403672 & BOLD:ACV1327 & Pingyang, Guizhou \\
\hline Protobothrops mucrosquamatus & $\mathrm{CH} 1$ & JX233625\# & - & Conghua, Guangdong \\
\hline Protobothrops trungkhanhensis & ZFMK 93774/CB 2012.53 & KP403673 & BOLD:ACV1573 & Vietnam \\
\hline Sinovipera sichuanensis & GP7 & KP403677 & BOLD:ACV0419 & Hejiang, Sichuan \\
\hline Sinovipera sichuanensis & GP2950 & KP403678 & BOLD:ACV0419 & Jiangkou, Guizhou \\
\hline Sinovipera sichuanensis & GP2952 & KP403679 & BOLD:ACV0419 & Jiangkou, Guizhou \\
\hline Trimeresurus albolabris & GP4 & KP403571 & BOLD:ABZ0912 & Hainan \\
\hline Trimeresurus albolabris & GP5 & KP403572 & BOLD:ABZ0912 & Hainan \\
\hline Trimeresurus albolabris & GP6 & KP403573 & BOLD:ABZ0912 & Fujian \\
\hline Trimeresurus albolabris & GP978 & KP403574 & BOLD:ABZ0912 & Cenxi, Guangxi \\
\hline Trimeresurus albolabris & GP979 & KP403575 & BOLD:ABZ0912 & Cenxi, Guangxi \\
\hline Trimeresurus albolabris & GP1591 & KP403576 & BOLD:ABZ0912 & Zhanjiang, Guangdong \\
\hline Trimeresurus albolabris & GP1592 & KP403577 & BOLD:ABZ0912 & Zhanjiang, Guangdong \\
\hline Trimeresurus albolabris & GP1895 & KP403578 & BOLD:ABZ0912 & Qinzhou, Guangxi \\
\hline
\end{tabular}




\begin{tabular}{|c|c|c|c|c|}
\hline Trimeresurus albolabris & GP1896 & KP403579 & BOLD:ABZ0912 & Qinzhou, Guangxi \\
\hline Trimeresurus albolabris & $\mathrm{H} 4$ & JX233622\# & - & Zhongshan, Guangdong \\
\hline Trimeresurus albolabris & ROM 30993 & KC291050\# & - & Hong Kong \\
\hline Trimeresurus albolabris & ROM 30854 & KC291060\# & - & Vinh Phuc, Vietnam \\
\hline Trimeresurus albolabris & ROM 30177 & KC291061\# & - & Vinh Phuc, Vietnam \\
\hline Trimeresurus albolabris & ROM 35301 & KC291062\# & - & Hia Duong, Vietnam \\
\hline Trimeresurus albolabris & ROM 35303 & KC291064\# & - & Hai Duong, Vietnam \\
\hline Trimeresurus albolabris & ROM 35299 & KC291065\# & - & Hai Duong, Vietnam \\
\hline Trimeresurus albolabris & ROM 35302 & KC291066\# & - & Hai Duong, Vietnam \\
\hline Trimeresurus albolabris & ROM 35300 & KC291067\# & - & Hai Duong, Vietnam \\
\hline Trimeresurus albolabris & ROM 37592 & KC291075\# & - & Kon Tum, Vietnam \\
\hline Trimeresurus albolabris & ROM 37595 & KC291077\# & - & Kon Tum, Vietnam \\
\hline Trimeresurus albolabris & ROM 18267 & KC291110\# & - & Vietnam \\
\hline Trimeresurus albolabris & ROM 30869 & KC291112\# & - & Vinh Phuc, Vietnam \\
\hline Trimeresurus albolabris & ROM 30856 & KC291113\# & - & Vinh Phuc, Vietnam \\
\hline Trimeresurus albolabris & ROM 30872 & KC291114\# & - & Vinh Phuc, Vietnam \\
\hline Trimeresurus albolabris & ROM 30867 & KC291115\# & - & Vinh Phuc, Vietnam \\
\hline Trimeresurus albolabris & ROM 30875 & KC291116\# & - & Vinh Phuc, Vietnam \\
\hline Trimeresurus albolabris & ROM 30864 & KC291117\# & - & Vinh Phuc, Vietnam \\
\hline Trimeresurus albolabris & ROM 30866 & KC291118\# & - & Vinh Phuc, Vietnam \\
\hline Trimeresurus albolabris & ROM 30871 & KC291119\# & - & Vinh Phuc, Vietnam \\
\hline Trimeresurus albolabris & ROM 30873 & KC291120\# & - & Vinh Phuc, Vietnam \\
\hline
\end{tabular}




\begin{tabular}{|c|c|c|c|c|}
\hline Trimeresurus albolabris & ROM 30861 & KC291121\# & - & Vinh Phuc, Vietnam \\
\hline Trimeresurus albolabris & ROM 30865 & KC291122\# & - & Vinh Phuc, Vietnam \\
\hline Trimeresurus albolabris & ROM 30863 & KC291124\# & - & Vinh Phuc, Vietnam \\
\hline Vipera berus & GP1390 & KP403681 & BOLD:AAW7158 & Jilin \\
\hline Vipera berus & NRM:6198 & KJ128676\# & - & Sweden \\
\hline Vipera renardi & GP634 & KP403684 & BOLD:ACV0623 & Nileike, Xinjiang \\
\hline Vipera renardi & - & KC122717\# & - & Kazakhstan \\
\hline Vipera renardi & - & KC122735\# & - & Kazakhstan \\
\hline Vipera renardi & - & KC122751\# & - & Crimea, Ukraine \\
\hline Vipera renardi & SNP 478 & KC122752\# & - & Astrakhan, Russia \\
\hline Vipera renardi & - & KC122753\# & - & Astrakhan, Russia \\
\hline Vipera renardi & - & KC122754\# & - & Crimea, Ukraine \\
\hline Vipera renardi & - & KC122759\# & - & Southern Kazakhstan \\
\hline Vipera renardi & - & KC122760\# & - & Adygea, Russia \\
\hline Vipera renardi & SNP 782-2 & KC122761\# & - & Kalmykia, Russia \\
\hline Viridovipera gumprechti & GP51 & KP403697 & BOLD:ACV0260 & Jingdong, Yunnan \\
\hline Viridovipera gumprechti & GP3271 & KP403698 & BOLD:ACS2952 & Daweishan, Yunnan \\
\hline Viridovipera gumprechti & GP3272 & KP403699 & BOLD:ACS2952 & Daweishan, Yunnan \\
\hline Viridovipera gumprechti & GP3273 & KP403700 & BOLD:ACS2952 & Daweishan, Yunnan \\
\hline Viridovipera gumprechti & GP3274 & KP403701 & BOLD:ACS2952 & Daweishan, Yunnan \\
\hline Viridovipera gumprechti & GP3275 & KP403702 & BOLD:ACS2952 & Daweishan, Yunnan \\
\hline Viridovipera gumprechti & GP3276 & KP403703 & BOLD:ACS2952 & Daweishan, Yunnan \\
\hline
\end{tabular}




\begin{tabular}{|c|c|c|c|c|}
\hline Viridovipera gumprechti & GP3286 & KP403704 & BOLD:ACS2952 & Daweishan, Yunnan \\
\hline Viridovipera gumprechti & $\mathrm{H} 17$ & KC171170\# & - & Honghe, Yunnan \\
\hline Viridovipera gumprechti & KIZ03525 & KP403705 & BOLD:ACS7018 & Jingdong, Yunnan \\
\hline Viridovipera stejnegeri & GP44 & KP403707 & BOLD:ACS2953 & Diaoluoshan, Hainan \\
\hline Viridovipera stejnegeri & GP45 & KP403708 & BOLD:ACS2953 & Diaoluoshan, Hainan \\
\hline Viridovipera stejnegeri & GP186 & KP403709 & BOLD:ACV0257 & Guilin, Guangxi \\
\hline Viridovipera stejnegeri & GP231 & KP403711 & BOLD:ACS2954 & Hejiang, Sichuan \\
\hline Viridovipera stejnegeri & GP342 & KP403712 & BOLD:ACS2954 & Shimen, Hunan \\
\hline Viridovipera stejnegeri & GP364 & KP403713 & BOLD:ACV1548 & Nanling, Guangdong \\
\hline Viridovipera stejnegeri & GP471 & KP403714 & BOLD:ACV1547 & Qimen, Anhui \\
\hline Viridovipera stejnegeri & GP472 & KP403715 & BOLD:ACV1547 & Qimen, Anhui \\
\hline Viridovipera stejnegeri & GP3300 & KP403716 & BOLD:ACS2954 & Kaiyuan, Yunnan \\
\hline Viridovipera stejnegeri & ROM 31133 & KC291052\# & - & Gia Lai, Vietnam \\
\hline Viridovipera stejnegeri & ROM 30954 & KC291053\# & - & Nghe An, Vietnam \\
\hline Viridovipera stejnegeri & ROM 30955 & KC291054\# & - & Nghe An, Vietnam \\
\hline Viridovipera stejnegeri & ROM 30956 & KC291055\# & - & Nghe An, Vietnam \\
\hline Viridovipera stejnegeri & ROM 30951 & KC291056\# & - & Nghe An, Vietnam \\
\hline Viridovipera stejnegeri & ROM 30952 & KC291057\# & - & Nghe An, Vietnam \\
\hline Viridovipera stejnegeri & ROM 30953 & KC291058\# & - & Nghe An, Vietnam \\
\hline Viridovipera stejnegeri & $\mathrm{H} 15$ & KC171168\# & - & Leishan, Guizhou \\
\hline Viridovipera stejnegeri & ROM 37590 & KC291071\# & - & Kon Tum, Vietnam \\
\hline Viridovipera stejnegeri & ROM 37591 & KC291072\# & - & Kon Tum, Vietnam \\
\hline Viridovipera stejnegeri & ROM 37596 & KC291073\# & - & Kon Tum, Vietnam \\
\hline
\end{tabular}




\begin{tabular}{lllll}
\hline Viridovipera stejnegeri & ROM 37594 & KC291074\# & - & Kon Tum, Vietnam \\
Viridovipera stejnegeri & ROM 37589 & KC291076\# & - & Kon Tum, Vietnam \\
Viridovipera stejnegeri & ROM 34567 & KC291084\# & - & Gia Lai, Vietnam \\
Viridovipera stejnegeri & ROM 27501 & KC291086\# & - & Kon Tum, Vietnam \\
Viridovipera stejnegeri & ROM 34667 & KC291088\# & - & Gia Lai, Vietnam \\
Viridovipera stejnegeri & ROM 34568 & KC291089\# & - & Gia Lai, Vietnam \\
Viridovipera stejnegeri & ROM 34565 & KC291090\# & - & Gia Lai, Vietnam \\
Viridovipera stejnegeri & ROM 34566 & KC291091\# & - & Gia Lai, Vietnam \\
Viridovipera stejnegeri & ROM 34554 & KC291099\# & - & Gia Lai, Vietnam \\
Viridovipera stejnegeri & ROM 30790 & KC291125\# & - & Gia Lai, Vietnam \\
Viridovipera stejnegeri & ROM 30791 & KC291126\# & - & Gia Lai, Vietnam \\
Viridovipera yunnanensis & GP 37 & KP403719 & BOLD:ACV0801 Huili, Sichuan \\
Viridovipera yunnanensis & GP 38 & KP403720 & BOLD:ACV0801 Huili, Sichuan \\
\hline
\end{tabular}

